\title{
The mechanism of human $\beta$-defensin 3 in MRSA-induced infection of implant drug-resistant bacteria biofilm in the mouse tibial bone marrow
}

\author{
CHEN ZHU, NI-RONG BAO, SHUO CHEN and JIAN-NING ZHAO \\ Department of Orthopaedic Surgery, Jinling Hospital, Nanjing University School of Medicine, \\ Nanjing, Jiangsu 210002, P.R. China
}

Received May 12, 2016; Accepted January 23, 2017

DOI: $10.3892 /$ etm.2017.4112

\begin{abstract}
The mechanism of human $\beta$-defensin 3 (HBD-3) in methicillin-resistant Staphylococcus aureus (MRSA-induced infection of implant drug-resistant bacteria biofilm in the mouse tibial bone marrow was studied. Healthy adult male Sprague-Dawley rats with average weight of $230 \mathrm{~g}$ were selected to construct the infection model of MRSA-induced implant drug-resistant bacteria biofilm in the mouse left tibial bone marrow.The drugs were intraperitoneally injected after $24 \mathrm{~h}$ medullary cavity infection, and the experimental groups included the model group, HBD-3 group, and vancomycin group (20 rats in each group). The model group was injected with $10 \mathrm{ml}$ saline, HBD-3 group was injected with $10 \mathrm{ml}$ of $8 \mu \mathrm{g} / \mathrm{ml}$ (1 MIC) and vancomycin group was injected with $10 \mathrm{ml}$ of $0.5 \mu \mathrm{g} / \mathrm{ml}$ (1 MIC), five animals in each group were sacrificed on the 1,7,14 and 21 days, respectively. Observation was carried out on whether there was swelling and purulent secretion on the local wound; $1 \mathrm{ml}$ venous sinus blood of eye socket was collected for blood routine examination and blood culture, and the laser scanning confocal microscopy was used to observe the morphology of the biofilm on the implant surface and the number of viable bacteria. Immunohistochemical staining was adopted to test the expression of nuclear factor $-\kappa \mathrm{B}(\mathrm{NF}-\kappa \mathrm{B})$ and toll-like receptor 4 (TLR-4), and ELISA method was used to test interleukin-10 (IL-10), tumor necrosis factor- $\alpha$ (TNF- $\alpha$ ), IL- $1 \alpha$ and interferon- $\gamma$ (INF- $\gamma$ )-inducible protein-10 (IP-10) expression levels. There was no death due to infection in the HBD-3 group or vancomycin group, 1 case with significant wound swelling was found, respectively, in each group, but
\end{abstract}

Correspondence to: Dr Jian-Ning Zhao, Department of Orthopaedic Surgery, Jinling Hospital, Nanjing University School of Medicine, 305 East Zhongshan Road, Nanjing, Jiangsu 210002, P.R. China

Email: zhaojianning.0207@163.com; jian_ningzhao1@163.com

Key words: human $\beta$-defensin 3, methicillin-resistant Staphylococcus aureus, biofilm, vancomycin, nuclear factor- $\kappa \mathrm{B}$, toll-like receptor 4 , interferon- $\gamma$-inducible protein there was no purulent secretion. The percentage of the total white blood cells and neutrophil granulocytes as well as the biofilm morphology and the number of viable bacteria in the model group was gradually increased with time, while those in the HBD-3 group and vancomycin group were decreased with time. The comparative difference among groups was statistically significant $(\mathrm{P}<0.05)$; those in the HBD-3 group and vancomycin group at each time-point was decreased significantly compared with the model group, and the difference among groups was statistically significant $(\mathrm{P}<0.05)$, but in terms of the comparison between the HBD-3 group and vancomycin group, the difference was not significantly different $(\mathrm{P}>0.05)$. The NF- $\kappa$ B and TLR-4 expressions in the model group and vancomycin group were not significantly changed at each time-point, those in the HBD-3 group began to increase on the 1st day, and reached the peak on the 7 th day and began to decline on the 14th day, and the comparative difference at each time-point was statistically significant $(\mathrm{P}<0.05)$; those in the HBD-3 group were significantly higher than the model group and vancomycin group at each time-point and the difference was statistically significant $(\mathrm{P}<0.05)$. The IL-10, TNF- $\alpha$, IL-1 $\alpha$, and IP-10 expressions in the model group at each time were significantly higher than the other two groups and the difference was statistically significant $(\mathrm{P}<0.05)$; in terms of the comparison between the HBD-3 group and vancomycin group, the difference was not statistically significant $(\mathrm{P}>0.05)$. In conclusion, $\beta$-defensin 3 can inhibit the bacterial growth by regulating inflammation and immune responses in the MRSAinduced implant drug-resistant bacteria biofilm infection in the mouse tibial bone marrow.

\section{Introduction}

The implant infection treatment is still problematic, and the new antibacterial drug endogenous antibiotic peptide, human $\beta$-defensin 3 (HBD-3), plays an important role in the inhibition of biofilm infection and inflammation as well as the immune regulation (1). The early in vitro studies found (2) that the killing role of HBD-3 in the methicillin-resistant Staphylococcus aureus (S. aureus) (MRSA) was significantly enhanced and it could inhibit the expression of the film formation gene icaAD and drug resistance gene $\mathrm{MecA}$; and after the stimulation 
of the $S$. aureus against the mouse osteoblasts, the mouse $\beta$-defensin-14 (MBD-14) congenetic to the osteoblast secreted HBD-3 in the structure and function could be promoted by activating the film-forming gene p38 mitogen-activated protein kinase (MAPK). In the models of MRSA-induced mouse acute suppurative osteomyelitis, the application of p38 MAPK agonist could promote MBD-14 at the infection site releasing the increased inhibition of the osteomyelitis (3). The present study further verified the influence of HBD-3 on the growth status and inflammatory response of the bacteria in the infectious biofilm as well as the cell cytokines in the infectious bone tissue of the implant in the mouse and provide the drug effect target and new theoretical basis for the clinical application of HBD-3.

\section{Materials and methods}

Construction of MRSA-induced implant drug-resistant bacteria biofilm infection model in the mouse left tibial bone marrow. Main reagents: S. aureus standard strain ATCC 25923 was purchased from the Bacterial Culture Laboratory of the Third Military Medical University, HBD-3 and vancomycin were purchased from Sigma-Aldrich (St. Louis, MO, USA), the nutrient broth ( $\mathrm{pH} 7.2+0.2$ ), tryptic soy agar (TSA) culture medium and tryptic soy broth (TSB) were purchased from Qingdao Haibo Biotechnology Co., Ltd. (Shandong, China); the nutrient agar was purchased from Hangzhou Microbiological Agents Co., Ltd. (Hangzhou, China), Live/Dead BacLight ${ }^{\mathrm{TM}}$ Bacterial Viability kit was purchased from Molecular Probes Europe BV (Leiden, The Netherlands); the calcofluor white was purchased from R\&D (Minneapolis, $\mathrm{MN}, \mathrm{USA})$. Mouse monoclonal nuclear factor $-\kappa \mathrm{B}(\mathrm{NF}-\kappa \mathrm{B})$ antibody (dilution, 1:500; cat. no. sc-56735), mouse monoclonal toll-like receptor 4 (TLR-4) antibody (dilution, 1:500; cat. no. sc-293072) and rabbit anti-mouse biotin-labeled secondary antibody (dilution, 1:2,000; cat. no. sc-358917) were purchased from Santa Cruz Biotechnology, Inc. (Santa Cruz, CA, USA). Diaminobenzidine (DAB) color-producing reagent kit was purchased from Beijing Zhongshan Golden Bridge Biotechnology Co., Ltd. (Beijing, China) and enzyme-linked immunosorbent assay (ELISA) kit was purchased from Invitrogen (Carlsbad, CA, USA).

Main instruments: The sterile super clean bench was purchased from Shanghai Boxun Industrial Co., Ltd. (Shanghai, China); the electro-heating standing-temperature cultivator type DHP-9162 was purchased from Beijing Liuyi Instrument Factory (Beijing, China), the laser scanning confocal microscope was purchased from Leica Microsystems Inc. (Buffalo Grove, IL, USA), the titanium rods (diameter, $0.5 \mathrm{~mm}$; length, $3 \mathrm{~mm}$ ) were processed and manufactured by Chongqing Lidakang Company (Chongqing, China), the Mindray BS-380 automatic biochemical analyzer was purchased from Shenzhen Mindray Bio-Medical Electronics Co., Ltd. (Shenzhen, China) and the ordinary optics microscope was purchased from Olympus (Tokyo, Japan).

The titanium rod was placed in 6-well flat bottom cell culture plate, the S. aureus was cultured in the TSA for $20 \mathrm{~h}$, after the culture, and a single colony was cultured in TSB for $20 \mathrm{~h}$ constantly shaking. The bacterial liquid was placed in each well of the 6-well plate, diluted with normal saline to $0.5 \mathrm{McIntosh}$ turbidity unit, each well was provided with $1 \mathrm{ml}$ TSB and the flat cells were cultured in the six wells, and then cultured in the incubator for $20 \mathrm{~h}$ at $37^{\circ} \mathrm{C}$. Healthy adult male Sprague-Dawley (SD) rats were selected under normal feeding, at the average weight of $230 \mathrm{~g}$. The mice were abdominally anesthetized and the medullary cavity was expanded by exposing the opening on the left-side tibial plateau, the titanium rods with biofilm were implanted in the medullary cavity, after $24 \mathrm{~h}$ medullary cavity infection, the drug was administered by intraperitoneal injection after the local biofilm was matured.

Experimental grouping and observation indicators. The cases in the experiment were divided into the model group, HBD-3 group and vancomycin group (20 in each group), the model group was injected with $10 \mathrm{ml}$ saline, HBD-3 group was injected with $10 \mathrm{ml}$ of $8 \mu \mathrm{g} / \mathrm{ml}$ (1 MIC), the vancomycin group was injected with $10 \mathrm{ml}$ of $0.5 \mu \mathrm{g} / \mathrm{ml}$ (1 MIC), 5 animals in each group were sacrificed on days $1,7,14$, and 21 , respectively, an observation was carried out on whether there was swelling and purulent secretion on the local wound, $1 \mathrm{ml}$ venous sinus blood of eye socket was collected for blood routine examination and blood culture, the laser scanning confocal microscopy was used to observe the morphology of the biofilm on the implant surface and the number of viable bacteria, the immunohistochemical staining was adopted to test the expression of NF- $\kappa \mathrm{B}$ and TLR-4, and ELISA method was used to test interleukin-10 (IL-10), tumor necrosis factor- $\alpha$ (TNF- $\alpha$ ), IL-1 $\alpha$ and interferon- $\gamma($ IFN- $\gamma)$-inducible protein-10 (IP-10) expression levels.

Laser scanning confocal microscope observation. After fluorescence staining, the polysaccharide protein complex became blue, a variety of bacteria on the bacterial biofilm was washed with phosphate-buffered saline (PBS), soaked in $2.5 \%$ glutaraldehyde solution for $1 \mathrm{~h}$, washed with PBS and added with calcofluor white, after $40 \mathrm{~min}$, they were wet, and confocal laser scanning microscopy (CLSM) was used to observe the biofilm morphology at 485/530 nm wavelength. The stain SYT09 in L13152 Live/Dead BacLight ${ }^{\mathrm{TM}}$ Bacterial Viability kits allowed viable bacteria to emit green fluorescence, and propidium iodide (PI) allow the dead bacteria to emit red fluorescence, the viable bacteria and dead bacteria was distinguished depending on the different fluorescence colors. The SYT09:PI:distilled water was prepared as 1.5:1.5:1 in the dark; the culture solution was removed; the deionized water was used for washing; the fluorescent dye was used for staining, and then the solution was incubated in the dark at $37^{\circ} \mathrm{C}$ for $15 \mathrm{~min}$ and placed under CLSM for testing; Image-Pro Plus version 6.0 (Media Cybernetics, Inc., Rockville, MD, USA) image analysis software was used for processing; three horizons were randomly selected, and finally the colony IOD, bulk density, and polysaccharide matrix staining area were calculated.

Immunohistochemical staining. The tibial tissue slices were conventionally manufactured through dewaxing, hydration, washing with PBS, enzyme inactivation with $3 \% \mathrm{H}_{2} \mathrm{O}_{2}$, antigen retrieval, $5 \%$ normal goat serum closure, declining of primary antibody working solution ( $\beta$-actin; dilution, 1:500; 
Table I. Comparison of the results of blood routine examination.

\begin{tabular}{lcccccr}
\hline & \multicolumn{7}{c}{ Groups } & & \\
\cline { 2 - 5 } Cells & Day & Model & HBD-3 & Vancomycin & F-value & P-value \\
\hline Total white & 1 & $10.6 \pm 1.8$ & $8.5 \pm 1.0$ & $8.8 \pm 1.2$ & 6.857 & 0.027 \\
blood cells (x10 $/ 1)$ & 7 & $12.2 \pm 2.0$ & $7.3 \pm 1.1$ & $7.5 \pm 1.3$ & 8.201 & 0.009 \\
& 14 & $14.5 \pm 2.2$ & $6.6 \pm 0.8$ & $6.7 \pm 1.0$ & 8.634 & 0.002 \\
F-value & 21 & $17.3 \pm 2.6$ & $5.0 \pm 0.6$ & $5.2 \pm 0.8$ & 9.125 & $<0.001$ \\
P-value & 9.538 & 7.628 & 7.549 & & & \\
Neutrophil & $<001$ & 0.012 & 0.013 & & & \\
granulocytes (\%) & 1 & $82.6 \pm 5.3$ & $78.9 \pm 4.6$ & $80.2 \pm 4.7$ & 7.322 & 0.026 \\
& 7 & $83.4 \pm 5.5$ & $75.3 \pm 4.4$ & $75.5 \pm 4.8$ & 7.564 & 0.023 \\
& 14 & $86.6 \pm 5.6$ & $73.4 \pm 4.2$ & $73.6 \pm 4.6$ & 7.747 & 0.020 \\
F-value & 21 & $88.0 \pm 6.0$ & $72.5 \pm 4.3$ & $72.6 \pm 4.5$ & 7.865 & 0.016 \\
P-value & 8.030 & 7.534 & 7.632 & & & \\
\hline
\end{tabular}

HBD-3, human $\beta$-defensin 3.

cat. no. sc-130300) and incubation at $37^{\circ} \mathrm{C}$ for $4 \mathrm{~h}$, washing with PBS, using appropriate amount of $50 \mu \mathrm{l}$ biotin-labeled secondary antibody working solution (dilution, 1:2,000; cat. no. sc-358917), incubation at $37^{\circ} \mathrm{C}$ for $30 \mathrm{~min}$ then washed with PBS, DAB developing, hematoxylin re-staining, dehydration, resulting in transparency and slice closure. The positive cells strained brown and yellow. The total cell count per slice was counted under complete horizon under high-power field (x400), three horizons were randomly selected, and then the mean value was taken.

Statistical analysis. SPSS 19.0 statistical software (SPSS, Inc., Chicago, IL, USA) was used for analysis; the quantitative data are expressed as mean \pm standard deviation; the comparison among the groups was analyzed by one-way ANOVA and the comparison among the groups was analyzed by variance requiring repeated data measurement; $\mathrm{P}<0.05$ was considered to indicate a statistically significant difference.

\section{Results}

General observations. Three mice in the model group died with swelling and white purulent secretion on the wound due to infection (15\%); no deaths occurred in the other two groups due to infection, and 1 case had significant wound swelling, respectively, in each group, but there was no purulent secretion.

Blood routine examination and blood culture. The percentage of the total white blood cells and neutrophil granulocytes in the model group was gradually increased with time, while that in the HBD-3 group and vancomycin group was decreased with time, and the comparative difference among groups was statistically significant $(\mathrm{P}<0.05)$; that in the HBD-3 group and vancomycin group at each time-point was significantly decreased compared with the model group, and the

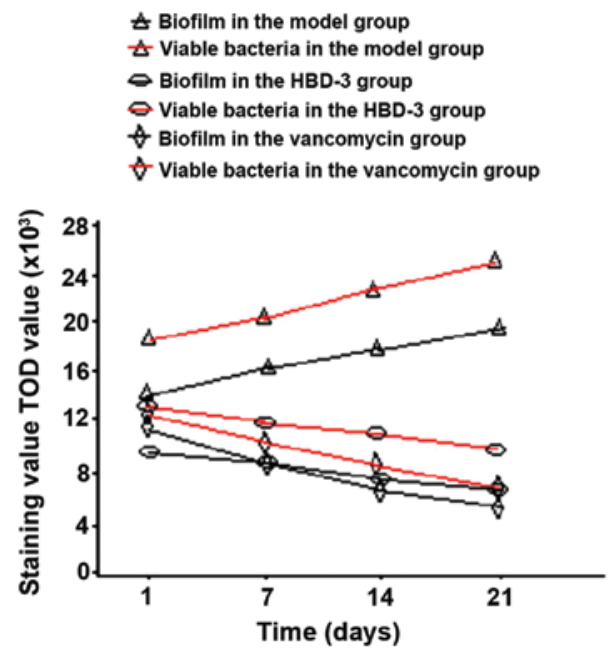

Figure 1. Comparison of biofilm morphology and the number of viable bacteria.

comparative difference was statistically significant (P-value between groups $<0.05$ ); in terms of the comparison between HBD-3 group and vancomycin group, the difference was not statistically significant $(\mathrm{P}>0.05)$ (Table I).

Through identification of the blood culture bacteria, the bacteria in the three groups were due to MRSA.

Biofilm morphology and the number of viable bacteria. The biofilm morphology and the number of viable bacteria in the model group were gradually increased with time, while those in the HBD-3 group and vancomycin group were decreased with time, the comparative difference among the groups was statistically significant $(\mathrm{P}<0.05)$, whereas, those in the HBD-3 group and vancomycin group at each time-point were significantly decreased compared with the model group, the comparative difference among groups was statistically significant $(\mathrm{P}<0.05)$; in terms of the comparison between HBD-3 

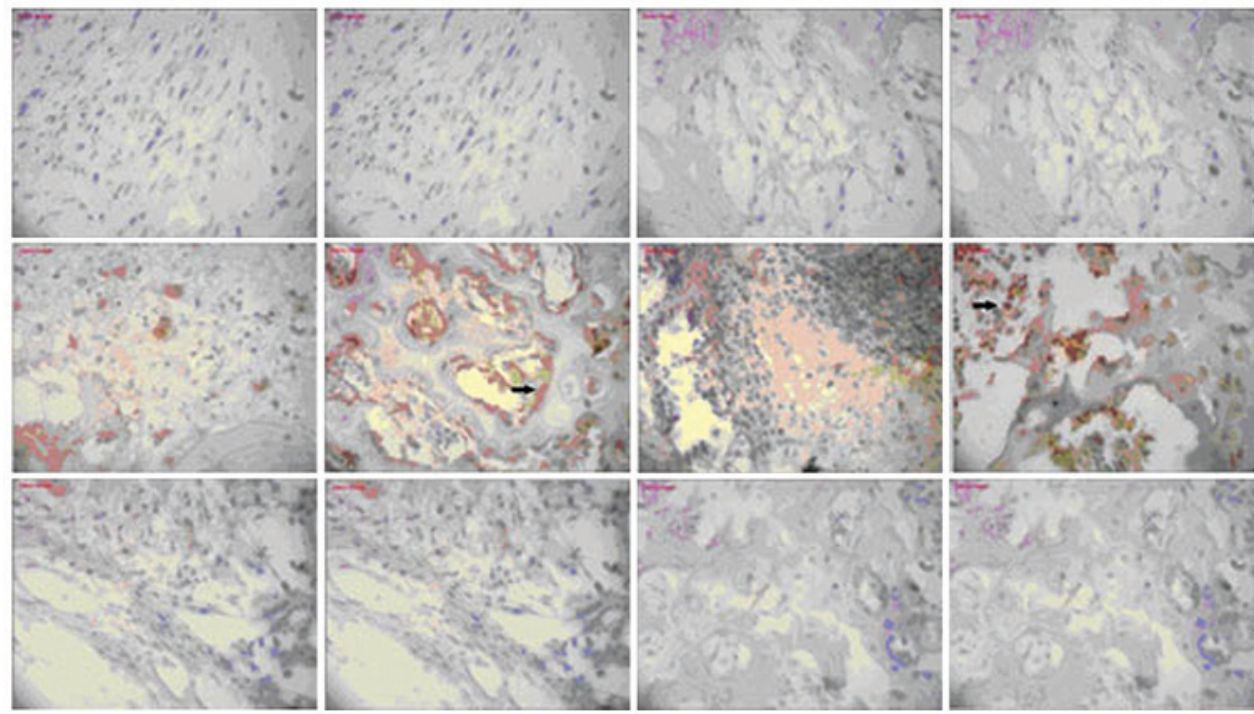

Figure 2. The expressions of NF-kB under immunohistochemical staining ( $\mathrm{x} 400$, from the left to right: 1, 7, 14 and 21 days in turn, and from top to bottom: The

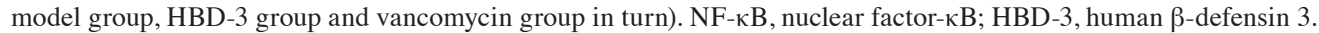
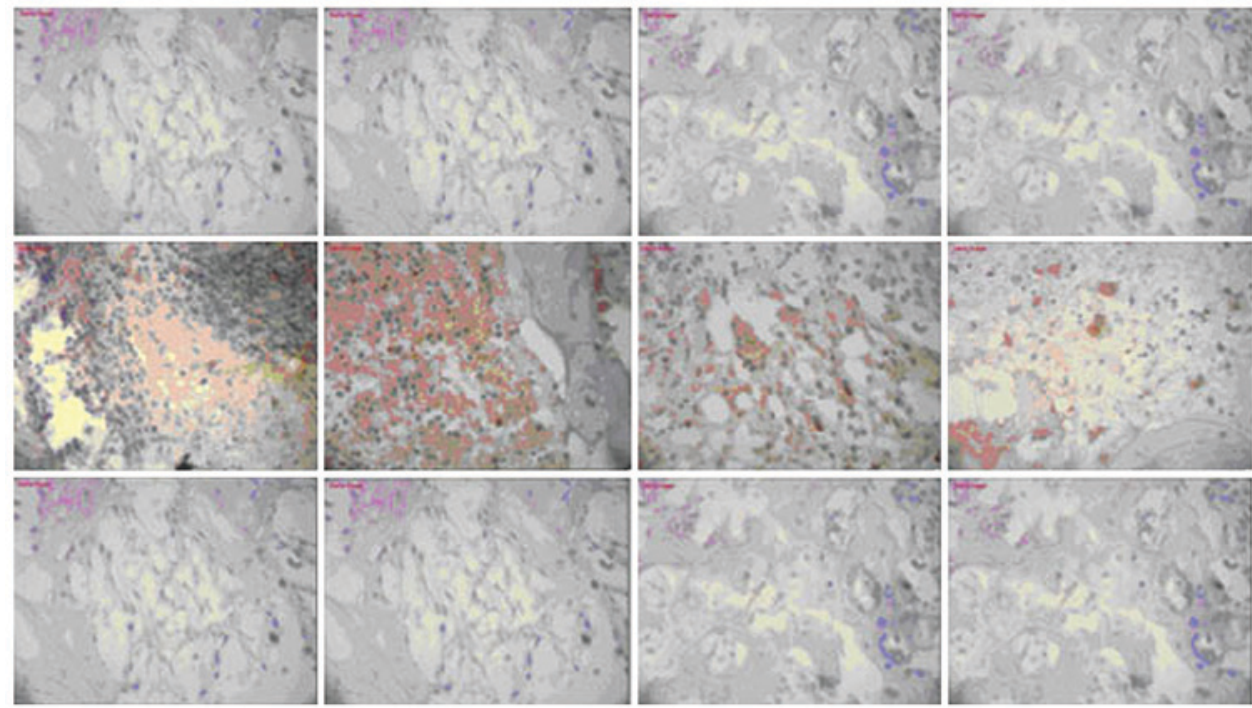

Figure 3. The expressions of TLR-4 under immunohistochemical staining ( $\mathrm{x} 400$, from left to right: 1, 7, 14 and 21 days, from top to bottom: The model group, HBD-3 group and vancomycin group). TLR-4, toll-like receptor 4; HBD-3, human $\beta$-defensin 3.

group and vancomycin group, the difference was not statistically significant $(\mathrm{P}>0.05)$ (Fig. 1).

Expression of $N F-\kappa B$ and TLR-4. There was no significant change in NF- $\kappa B$ or TLR-4 expression in the model group or vancomycin group at each time-point, those in the HBD-3 group began to increase on the 1st day, reached the peak on the 7th day and began to decrease on the 14th day, the comparative difference at each time-point was statistically significant $(\mathrm{P}<0.05)$, whereas, those in the HBD-3 group at each time-point were significantly higher than the model group and vancomycin group, and the difference was statistically significant $(\mathrm{P}<0.05)$ (Figs. 2 and 3$)$.

The expression levels of IL-10, TNF- $\alpha, I L-1 \alpha$ and IP-10. The expression levels of IL-10, TNF- $\alpha$, IL-1 $\alpha$ and IP-10 at each time-point in the model group were significantly higher than the other two groups, and the difference was statistically significant $(\mathrm{P}<0.05)$; in terms of the comparison between the HBD-3 group and vancomycin group, the difference was not statistically significant $(\mathrm{P}>0.05)$ (Fig. 4).

\section{Discussion}

A previous study (4) has shown that after bacterial infection, the osteoblasts could be stimulated to secret cytokines, chemokines, and endogenous antibiotic peptide $\beta$-defensin, suggesting that in addition to the inherent bone metabolism regulation function, the osteoblast could also help active immune and defense response and acquired immune regulation of the host. While under the bacterial inflammation condition, the $\beta$-defensins secreted by the osteoblasts, as a kind of inhibitor 
A

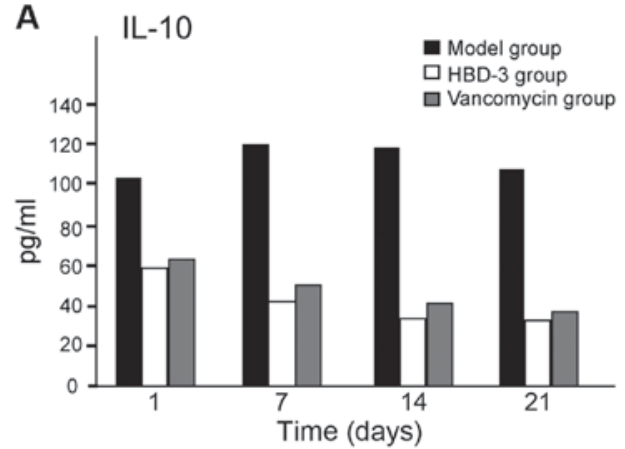

C

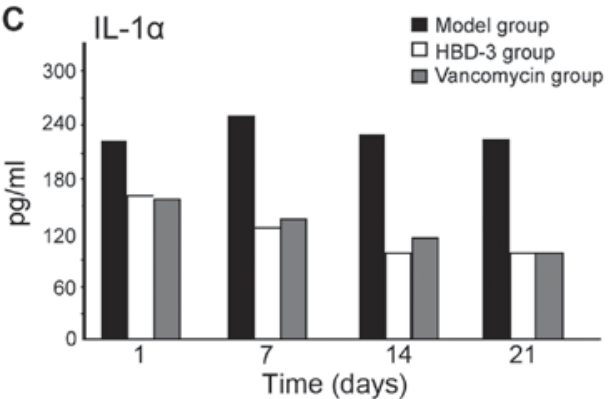

B
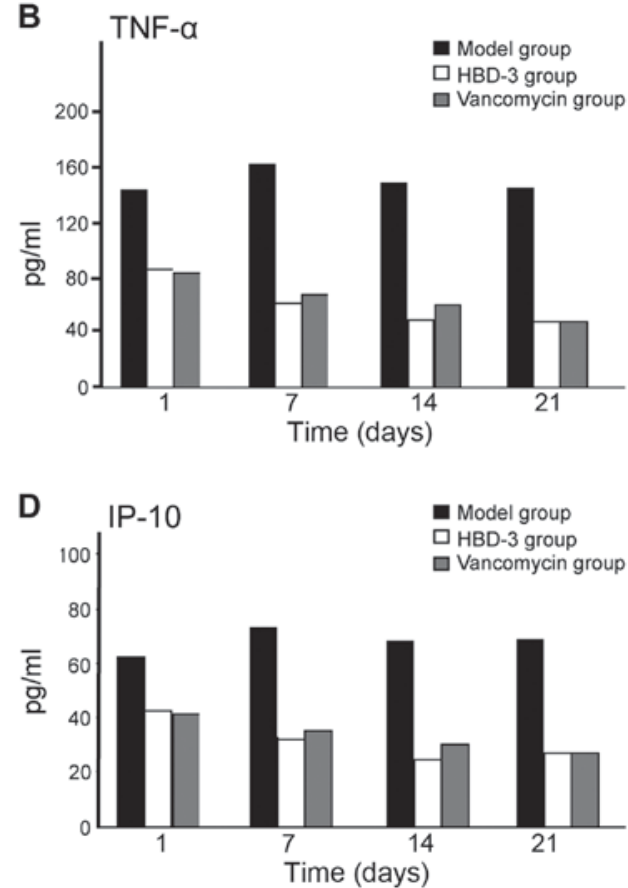

Figure 4. Four expression levels of (A) IL-10, (B) TNF- $\alpha$, (C) IL-1 $\alpha$ and (D) IP-10. IL-10, interleukin-10; TNF- $\alpha$, tumor necrosis factor- $\alpha$; IP-10, inducible protein-10.

for bacterial infection newly discovered in recent years, are attracting increasing attention in clinical and basic research because of its broad antibacterial spectrum, greater action speed, the relative difficulty of bacterial mutation resistance to the drug, inherent immunological compatibility and special role in bone infection immune regulation and control (5).

$\beta$-defensin, as a kind of cationic protein polypeptide with the molecular weight of 2-6 kDa, has potent antimicrobial activity (6) if the concentration is from $\mu \mathrm{mol}$ to $\mathrm{nmol}$. Compared with other antimicrobial peptides, HBD-3 has a broad antibacterial spectrum, stable physical and chemical properties as well as a strong bactericidal effect on the Gram-positive, negative and fungi. It can pass through the mediation of TLR and connect with congenital and acquired immune responses (7). The amino acid sequence of the MBD-14 is highly congenetic (8) to that of HBD-3. Furthermore, the in vitro antibacterial experiments and immune chemotaxis experiments have also showed (9) that the recombinant MBD-14 has a similar broad-spectrum antibacterial and chemotactic cytokine activity with HBD-3. $\beta$-defensin-class antibacterial mechanism may be due to the peptide molecules with cationic charges that can be combined first on the bacterial plasma membrane with the cationic charge, then the hydrophobic segments and amphipathic $\alpha$-helixes in the molecules will be inserted into the plasma membrane, eventually the ion channel can be formed by mutual displacement of the membrane molecules, thereby resulting in bacterial membrane structural damage and causing bacteria death (10) due to loss of membrane potential. Since the $\beta$-defensin-class antibacterial mechanism is mainly a physical effect, it is significantly different from the mechanism of traditional antibiotics. Therefore, it is difficult for microorganism to produce resistance mutation against it, this is the reason that $\beta$-defensins can still be provided with a bactericidal effect on many drug-resistant bacteria even including resistance to the vancomycin Enterococcus faecalis, and it will not damage its own cells and visceral organs (11).

Our study results show that there was no death due to infection in the HBD-3 group and vancomycin group, there was only one case with significant wound swelling in the two groups, but there were no purulent secretions, suggesting that HBD-3 and vancomycin had a strong antibacterial activity. The percentage of the total white blood cells and the neutrophil granulocytes in the HBD-3 group and vancomycin groups as well as the biofilm morphology and the number of viable bacteria were decreased with time, those at each time-point were significantly decreased compared with the model group, the comparative differences among the groups and in the group were statistically significant; in terms of the comparison between the HBD-3 group and vancomycin group, the difference was not statistically significant. It was suggested that HBD-3 and vancomycin had a strong bacteriostatic effect on the bacterial growth and biofilm formation and the bacteriostatic effects were equivalent (12). The expression of NF- $\kappa \mathrm{B}$ and TLR-4 in the model group and vancomycin group was not significantly changed at each time-point, those in the HBD-3 group began to increase on the 1st day, reached the peak on the 7th day and began to decline on the 14th day, those in the HBD-3 group at each time-point were significantly higher than the model group and the vancomycin group and the difference was statistically significant. It was suggested that the bacteriostatic effect of HBD-3 may be related to the upregulation of the expressions of $\mathrm{NF}-\kappa \mathrm{B}$ and TLR-4 (13), while the bacteriostatic effect of vancomycin may be accomplished by other means. The expression levels of IL-10, TNF- $\alpha$, IL-1 $\alpha$ and IP-10 in the model group at each time-point were significantly higher than the other two groups, 
and the difference was statistically significant; in terms of the comparison between the HBD-3 group and vancomycin group, the difference was not statistically significant. It was suggested that HBD-3 and vancomycin could significantly reduce the IL-10, TNF- $\alpha$, IL- $1 \alpha$ and IP-10 mediated inflammatory response (14). In conclusion, $\beta$-defensin 3 can inhibit the bacterial growth by regulating inflammation and immune response in MRSA-induced implant drug-resistant bacteria biofilm infection in mouse tibial bone marrow.

\section{Acknowledgements}

This study was supported by the National Natural Science Foundation of China (grant no. 81401815), the China Postdoctoral Science Foundation (grant no. 2015M582900) and the Jiangsu Postdoctoral Science Foundation (grant no. 1501146 C).

\section{References}

1. Montanaro L, Speziale P, Campoccia D, Ravaioli S, Cangini I, Pietrocola G, Giannini S and Arciola CR: Scenery of Staphylococcus implant infections in orthopedics. Future Microbiol 6: 1329-1349, 2011.

2. Batoni G, Maisetta G, Esin S and Campa M: Human beta-defensin-3: a promising antimicrobial peptide. Mini Rev Med Chem 6: 1063-1073, 2006.

3. Shi Y, Song W, Feng ZH, Zhao YT, Li F, Tian Y and Zhao YM: Disinfection of maxillofacial silicone elastomer using a novel antimicrobial agent: recombinant human beta-defensin-3. Eur J Clin Microbiol Infect Dis 28: 415-420, 2009.

4. Zhu C, Tan H, Cheng T, Shen H, Shao J, Guo Y, Shi S and Zhang X: Human $\beta$-defensin 3 inhibits antibiotic-resistant Staphylococcus biofilm formation. J Surg Res 183: 204-213, 2013.

5. Zhu C, He N, Cheng T, Tan H, Guo Y, Chen D, Cheng M, Yang Z and Zhang X: Ultrasound-targeted microbubble destruction enhances human $\beta$-defensin 3 activity against antibiotic-resistant Staphylococcus biofilms. Inflammation 36: 983-996, 2013.
6. Singh PK, Shiha MJ and Kumar A: Antibacterial responses of retinal Müller glia: production of antimicrobial peptides, oxidative burst and phagocytosis. J Neuroinflammation 11: 33, 2014.

7. Colavita I, Nigro E, Sarnataro D, Scudiero O, Granata V, Daniele A, Zagari A, Pessi A and Salvatore F: Membrane protein $4 \mathrm{~F} 2 / \mathrm{CD} 98$ is a cell surface receptor involved in the internalization and trafficking of human $\beta$-Defensin 3 in epithelial cells. Chem Biol 22: 217-228, 2015.

8. Hinrichsen K, Podschun R, Schubert S, Schröder JM, Harder J and Proksch E: Mouse beta-defensin-14, an antimicrobial ortholog of human beta-defensin-3. Antimicrob Agents Chemother 52: 1876-1879, 2008

9. Röhrl J, Yang D, Oppenheim JJ and Hehlgans T: Identification and biological characterization of mouse beta-defensin 14 , the orthologue of human beta-defensin 3. J Biol Chem 283: 5414-5419, 2008 .

10. Bedran TB, Mayer MP, Spolidorio DP and Grenier D: Synergistic anti-inflammatory activity of the antimicrobial peptides human beta-defensin-3 (hBD-3) and cathelicidin (LL-37) in a three-dimensional co-culture model of gingival epithelial cells and fibroblasts. PLoS One 9: e106766, 2014.

11. Semple F, MacPherson H, Webb S, Cox SL, Mallin LJ, Tyrrell C, Grimes GR, Semple CA, Nix MA, Millhauser GL, et al: Human $\beta$-defensin 3 affects the activity of pro-inflammatory pathways associated with MyD88 and TRIF. Eur J Immunol 41: 3291-3300, 2011.

12. Funderburg N, Lederman MM, Feng Z, Drage MG, Jadlowsky J, Harding CV, Weinberg A and Sieg SF: Human-defensin-3 activates professional antigen-presenting cells via toll-like receptors 1 and 2. Proc Natl Acad Sci USA 104: 18631-18635, 2007.

13. Ning R, Zhang X, Guo X and Li Q: Staphylococcus aureus regulates secretion of interleukin- 6 and monocyte chemoattractant protein-1 through activation of nuclear factor kappaB signaling pathway in human osteoblasts. Braz J Infect Dis 15: 189-194, 2011.

14. Zhu C, Wang J, Cheng T, Li Q, Shen H, Qin H, Cheng M and Zhang X: The potential role of increasing the release of mouse $\beta$-defensin-14 in the treatment of osteomyelitis in mice: a primary study. PLoS One 9: e86874, 2014. 